Journal of Systems Science and Information

Oct., 2016, Vol. 4, No. 5, pp. 444-459

DOI: $10.21078 /$ JSSI-2016-444-16

\title{
AEWMA $t$ Control Chart for Short Production Runs
}

\author{
Zhiyuan CHANG \\ School of Automation, Nanjing University of Science and Technology, Nanjing 210064, China; School \\ of Information Engineering, Southwest University of Science and Technology, Mianyang 621010, \\ China \\ E-mail: changzy0510@163.com \\ Jinsheng SUN \\ School of Automation, Nanjing University of Science and Technology, Nanjing 210064, China \\ E-mail: jssun67@163.com
}

\begin{abstract}
Owing to the limited number of inspections during a short run process, it is impossible to get the correct estimate of the population mean and standard deviation during Phase I implementation of control chart. The $t$ control chart proposed recently can overcome this problem. The EWMA $t$ control chart has been proposed to monitor the process mean, but a single EWMA $t$ control chart cannot perform well for small and large shifts simultaneously, which is known as the "inertia problem". The adaptive varying smoothing parameter EWMA (AEWMA) control chart can overcome the inertia problem. In this paper, the AEWMA $t$ control chart for short run process is proposed. The truncated average run length and the probability of trigger a signal are adopted to test the performance of short run AEWMA $t$ chart. Based on the investigation of the joint effect of control chart parameters on the performance of AEWMA $t$ chart, a new optimization algorithm is proposed for statistical design of the AEWMA control chart. Simulations are performed for perfect and imperfect setup conditions, the results show that the AEWMA $t$ control chart performs better than the EWMA $t$ control chart.
\end{abstract}

Keywords AEWMA control chart; $t$ chart; short run; TARL; Markov chain

\section{Introduction}

Control charts are effective tools in statistical process control (SPC) for process monitoring and quality improvement. Usually, SPC manuals associate the Shewhart, EWMA and CUSUM control charts implementation with manufacturing processes which operate indefinitely. In fact, resource capacity within manufacturing systems characterized by a high degree of flexibility and variety of produced items is usually scheduled to manufacture short runs of productions. This kind of phenomenon is common in job shop manufacturing of small series of mechanical parts. Because the traditional control charts have to assume that the process mean and deviation are known previously or estimate the process parameters by taking many samples, the short run property leads to many difficulties in implementing control charts, such as there are not enough samples to estimate distribution parameters.

Received August 26, 2015, accepted November 10, 2015

Supported by the National Natural Science Foundation of China (70931002) 
Usually, at least $25 \sim 30$ samples having size $n$ are taken in Phase I control chart to correctly estimate the distribution parameters of the monitored statistic. In particular, to get estimated limits close to the true chart limits, Quesenberry ${ }^{[1]}$ has suggested that at least $400 /(n-1)$ samples should be taken. Short production runs allowing for a limited number of scheduled inspections, results in a number of samples which is too small. To overcome this problem, many researchers have investigated the procedure to reduce the samples in Phase I chart. Neduraman and Pigniatiello ${ }^{[2]}$ and Tsai et al. ${ }^{[3,4]}$ have proposed different procedures based on evaluating statistics having a $t$ distribution to construct the prospective control limits with a few initial subgroups. These procedures still need a few of initial subgroups, even the initial sample numbers are decreased; thus, they are more effect in medium or large run production. Quesenberry ${ }^{[5,6]}$ has proposed self-starting charts by defining a set of sequential $Q$ statistics to detect the shifts in process mean or variance. Castillo and Montgomery ${ }^{[7]}$ have proposed some enhancements for $Q$ chart: An exponentially weighted moving average (EWMA) method and an adaptive Kalman filtering method. He, et al. ${ }^{[8]}$ has indicated that $Q$ charts are biased with unknown variances. For some specific mean shift, the $Q$ charts have a large out-of-control average run length than the expected number of false alarms; this problem is particularly obvious if the shift occurs at the early stage of sampling, which is known as 'masking of the shift' problem. Celano, et al. ${ }^{[9]}$ have demonstrated that the modified $Q$ charts proposed by He, et al. ${ }^{[8]}$ still present some bias and difficulty of implementation in practice.

Recently, $t$ control chart has been proposed by Zhang, et al. ${ }^{[10]}$, and the performances of Shewhart $t$ chart and EWMA $t$ chart for long runs have been investigated against the errors in estimating the process standard deviation. Kazemzadeh ${ }^{[1]}$ have investigated the variable sampling interval (VSI) EWMA $t$ chart. As an efficient quality control tool for short production runs, $t$ chart has been investigated as a self-starting control chart. Nenes and Tagaras ${ }^{[12]}$ have proposed two proper statistical performance measurement. One of them is truncated average run length (TARL), which is defined as the mean number of samples until a signal or until the completion of the process, whichever occurs first. The other on is the probability of having at least one signal during an out-of-control production run. Utilizing these measures, Celano ${ }^{[9]}$ have investigated the short run Shewhart $t$ chart and EWMA $t$ chart with perfect and imperfect set-up conditions, and the self-starting property of $t$ chart has been investigated. Celano ${ }^{[13]}$ have investigated the performance of Shewhart, EWMA and CUSUM $t$ charts with unknown shift size. Besides, the economic performances of Shewhart and CUSUM $t$ chart for short production runs have been investigated by Celano ${ }^{[14,15]}$, and the economic model for short production runs has been specifically designed.

However, a single EWMA control chart can not perform well for small and large shifts simultaneously ${ }^{[16]}$. The small size of smoothing parameter $\lambda$ will lead to the potential delay in detecting sudden large shift, which is known as the 'inertia problem'. To overcome the 'inertia problem' of EWMA control chart, the adaptive varying smoothing parameter EWMA (AEWMA) control chart that weights the past observations of the monitored process using a suitable function of current error has been proposed by Capizzi and Masarotto ${ }^{[16]}$. Woodall and Mahmoud ${ }^{[17]}$ have defined a new measure of inertia, the signal resistance (SR). The value of SR shows that AEWMA chart has much better inertial properties than the ordinary EWMA 
chart. Shu ${ }^{[18]}$ has investigated the property of AEWMA control chart for monitoring process dispersion, and proposed another optimization method for AEWMA control chart. Saleh, et al. ${ }^{[19]}$ have denoted that the AEWMA chart outperform the EWMA chart to monitor the process mean when process parameters are estimated, especially when a small number of Phase I samples is available and the shift size is unpredictable.

To overcome the inertia problem of EWMA $t$ control chart, we propose an AEWMA $t$ control chart for short production runs in this paper. The Markov chain method is implemented to calculate the TARL of AEWMA $t$ control chart. The joint effect of parameters on the TARL performance is investigated. Based on the investigation, a new optimization algorithm of AEWMA control chart is proposed. The simulation results show that the performance of AEWMA $t$ control chart is superior to the EWMA $t$ control chart.

The rest of paper is organized as follow. In Section 2, the monitored $T$ statistic is introduced. In Section 3, the AEWMA $t$ control chart is proposed, and the Markov chain methods used to for computing the statistical measures are developed. In Section 4, optimization statistical design procedure of AEWMA $t$ control chart are introduced based in the analysis of joint effects of parameters $\lambda$ and $\gamma$. In Section 5, considering the two property measures, the performance of AEWMA $t$ control chart is compared with EWMA $t$ control chart. Finally, some conclusions are given.

\section{The $T$ Statistic}

Assuming that, $N$ parts during a production horizon $H$ are scheduled to produce in a manufacturing process. The normal distributed quality character $X \sim N\left(\mu_{0}+\delta \sigma_{0}, \tau \sigma_{0}\right)$ with the target value equaling to $M$ should be monitored, where $\mu_{0}$ and $\sigma_{0}$ are the in-control population mean and standard deviation, respectively. The shift of the mean and deviation are measured as the multiples $\delta$ and $\tau$ of the in-control population standard deviation $\sigma_{0}$, respectively. Let Is denotes the number of scheduled inspections within the production horizon $H$. A subgroup $\left\{X_{i, 1}, X_{i, 2}, \cdots, X_{i, n}\right\}, i=1,2, \cdots, I s$ of $n$ measures is collected at $i$ th inspection epoch. The sample size $n$ is subject to $n \leq N / I s$. The subgroup mean $\bar{X}_{i}$ and standard deviation $S_{i}$ are computed as:

$$
\begin{aligned}
\bar{X}_{i} & =\frac{1}{n} \sum_{j=1}^{n} X_{i, j}, \\
S_{i} & =\sqrt{\frac{1}{n-1} \sum_{j=1}^{n}\left(X_{i, j}-\bar{X}_{i}\right)^{2}} .
\end{aligned}
$$

At the beginning of the production, the in-control mean $\mu_{0}$ should be as close as possible to the target value $M$. However, the process begin with an initial setup error is common. The setup error is measured as the multiple $\Delta$ of $\sigma_{0}$. That is to say, $\Delta=0$ denotes that the process is perfect setup, $\mu_{0}=M$; otherwise, an initial setup error $\Delta \sigma_{0}$ will shift the in-control population mean $\mu_{0}=M$ to $\mu_{0}=M+\Delta \sigma_{0}$. When parameter values are $\Delta \neq 0, \delta \neq 0$ and $\tau>1$, the statistic $T_{i}$ can be computed:

$$
T_{i}=\frac{\bar{X}_{i}-M}{S_{i} / \sqrt{n}}
$$




$$
\begin{aligned}
& =\frac{\sqrt{n}\left(\bar{X}_{i}-M\right)+\mu_{1} \sqrt{n}-\mu_{1} \sqrt{n}}{S_{i}} \\
& =\frac{\sqrt{n}\left(\bar{X}_{i}-\mu_{1}\right) / \sigma_{1}+\sqrt{n}\left(\mu_{0}+\delta \sigma_{0}-\mu_{0}+\Delta \sigma_{0}\right) / \sigma_{1}}{S_{1} / \sigma_{1}} \\
& =\frac{Z+\sqrt{n}(\delta+\Delta) / \tau}{\sqrt{\frac{V}{n-1}}},
\end{aligned}
$$

where $\sigma_{1}=\tau \cdot \sigma_{0}$ represents the out-of-control process deviation; $Z=\sqrt{n}\left(\bar{X}_{i}-\mu_{1}\right) / \sigma_{1}$ is a standard normal random variable; $V=(n-1)\left(S_{i} / \sigma_{1}\right)^{2}$ is chi-squared distributed with $n-1$ degrees of freedom. The standard deviation of $T_{i}$ is:

$$
\sigma_{T}=\sqrt{\frac{n-1}{n-3}}
$$

where $n$ should be greater than 3 . The process is in-control meaning that $\Delta=0, \delta=0$ and $\tau=1$, the statistic $T_{i}$ obeys $t$ distribution. We define the notation $F(\cdot \mid \nu)$ as the $t$ cumulative distribution function with $\nu$ degrees of freedom. Otherwise, when the process is out-of-control, the statistic $T_{i}$ obeys a non-central $t$ distribution for $\Delta \neq 0$ or $\delta \neq 0$. We define the notation $G(\cdot \mid \nu, \psi)$ as the non-central $t$ cumulative distribution function with $\nu$ degrees of freedom, and $\psi$ is the non-central parameter.

\section{AEWMA $t$ Control Chart}

The EWMA $t$ control chart has been investigated by many researchers with the form:

$$
Y_{i}=\lambda T_{i}+(1-\lambda) Y_{i-1}, \quad Y_{0}=0,
$$

where $\lambda \in(0,1]$ is the smoothing constant. For EWMA $t$ chart, the smoothing parameter $\lambda$ will remain the same during the whole process. The small value of smoothing parameter $\lambda$ will lead to large potential delay in detecting a sudden large shift, which is known as the "inertia problem". To overcome the inertial problem, Capizzi and Masarotto ${ }^{[16]}$ have proposed an alternative form of EWMA control chart, which is known as AEWMA chart. We utilize the AEWMA chart to monitor the $T$ statistic, which is defined as AEWMA $t$ chart. The AEWMA $t$ chart is described as:

$$
Y_{i}=Y_{i-1}+\lambda\left(T_{i}-Y_{i-1}\right)=Y_{i-1}+\phi\left(e_{i}\right), \quad Y_{0}=\mu_{0},
$$

where $e_{i}=T_{i}-Y_{i-1}$ is the estimation error of $i$ th epoch; $\phi\left(e_{i}\right)$ is a score function. By defining $\omega\left(e_{i}\right)=\phi\left(e_{i}\right) / e_{i}$, the equation (6) can be rewritten as follow:

$$
Y_{i}=\omega\left(e_{i}\right) T_{i}+\left(1-\omega\left(e_{i}\right)\right) Y_{i-1}, \quad Y_{0}=\mu_{0}
$$

[16] has given three score functions, the function $\phi_{h u}(e)$ has been widely used ${ }^{[18,19,21]}$. The score function $\phi_{h u}(e)$ is defined as follow:

$$
\phi_{h u}(e)= \begin{cases}e+(1-\lambda) k, & e<-k, \\ \lambda e, & |e| \leq k, \\ e-(1-\lambda) k, & e>k .\end{cases}
$$


The control limits of AEWMA $t$ chart can be defined as the multiple of $\sigma_{T}$. The control limits of AEWMA $t$ control chart equal to:

$$
U C L=h, \quad L C L=-h, \quad h=L \delta_{T} \sqrt{\frac{\lambda}{2-\lambda}} .
$$

A useful statistic measure of control chart is the average run length (ARL), which is defined as the expected number of samples until the chart triggers a signal. For the short run case, where Is is limited, the process may end without the chart having issued any out-of-control signal. Therefore, truncated ARL (TARL) is a proper statistical measure for short production runs. If the process ends without any signal during the $I s$ samples, the truncated run length is $I s+1$.

To calculate the TARL of AEWMA $t$ control chart, the Markov chain theory is implemented. The interval from $L C L$ to $U C L$ is partitioned into $2 m+1$ subintervals $I_{j}, j=-m, \cdots, 0, \cdots, m$. The width of each subinterval is $d=(U C L-L C L) /(2 m+1)$. The statistic $Y_{i}$ is said to be in state $j$ at time $i$ which means that the inequality $S_{j}-d / 2<Y_{i}<S_{j}+d / 2$ is satisfied, where $S_{j}$ is the midpoint of subinterval $I_{j}$. Assuming that $Y_{i}=S_{j}$, whenever it falls in subinterval $I_{j}$. The absorbing state is denoted as $m+1$ state, which means that $Y_{i}<L C L$ or $Y_{i}>U C L$. The transition probability matrix $P$ is as follow:

$$
P=\left[\begin{array}{cccccc}
p_{m,-m} & \cdots & p_{m, 0} & \cdots & p_{m, m} & p_{m, m+1} \\
\vdots & \vdots & \vdots & \vdots & \vdots & \vdots \\
p_{0,-m} & \cdots & p_{0,0} & \cdots & p_{0, m} & p_{0, m+1} \\
\vdots & \vdots & \vdots & \vdots & \vdots & \vdots \\
p_{-m,-m} & \cdots & p_{-m, 0} & \cdots & p_{-m, m} & p_{-m, m+1} \\
0 & \cdots & 0 & \cdots & 0 & 1
\end{array}\right]=\left[\begin{array}{cc}
A_{(2 m+1,2 m+1)} & B_{(2 m+1,1)} \\
0_{(1,2 m+1)} & 1
\end{array}\right]
$$

where the generic element $p_{j, k}$ of $P$ represents the probability that statistic $Y_{i}$ goes from state $j$ to state $k$ in one step. The value of $p_{j, k}$ can be calculated:

$$
\begin{aligned}
p_{j, k} & =\operatorname{Pr}\left(S_{k}-d / 2<Y_{i}<S_{k}+d / 2 \mid Y_{i-1}=S_{j}\right) \\
& =\operatorname{Pr}\left(S_{k}-d / 2<Y_{i-1}+\phi_{h u}\left(T_{i}-Y_{i-1}\right)<S_{k}+d / 2 \mid Y_{i-1}=S_{j}\right) \\
& =\operatorname{Pr}\left(S_{j}+\phi_{h u}^{-1}\left(S_{k}-S_{j}-d / 2\right)<T_{i}<S_{j}+\phi_{h u}^{-1}\left(S_{k}-S_{j}+d / 2\right)\right) \\
& =F\left(S_{j}+\phi_{h u}^{-1}\left(S_{k}-S_{j}+d / 2\right) \mid n-1\right)-F\left(S_{j}+\phi_{h u}^{-1}\left(S_{k}-S_{j}-d / 2\right) \mid n-1\right),
\end{aligned}
$$

where $\phi_{h u}^{-1}(v)$ is the inverse function of $\phi_{h u}(e)$. The form of $\phi_{h u}^{-1}(v)$ is

$$
\phi_{h u}^{-1}(v)= \begin{cases}v-(1-\lambda) \gamma, & v<-\lambda \gamma, \\ v / \lambda, & |v|<\lambda \gamma, \\ v+(1-\lambda) \gamma, & v>\lambda \gamma,\end{cases}
$$

where, $\lambda$ and $\gamma$ are two decision parameters of AEWMA chart.

The value of $p_{j, m+1}$ denotes that $Y_{i}$ goes from in-control state $j$ to the absorbing state $m+1$, and $p_{j, m+1}$ can be calculated:

$$
p_{j, m+1}=\operatorname{Pr}\left(Y_{i}<L C L \mid Y_{i-1}=S_{j}\right) \cup \operatorname{Pr}\left(Y_{i}>U C L \mid Y_{i-1}=S_{j}\right)
$$




$$
\begin{aligned}
& =\operatorname{Pr}\left(T_{i}<\phi_{h u}^{-1}\left(L C L-S_{j}\right)+S_{j}\right)+\operatorname{Pr}\left(T_{i}>\phi_{h u}^{-1}\left(U C L-S_{j}\right)+S_{j}\right) \\
& =F\left(\phi_{h u}^{-1}\left(L C L-S_{j}\right)+S_{j}\right)+1-F\left(\phi_{h u}^{-1}\left(U C L-S_{j}\right)+S_{j}\right) .
\end{aligned}
$$

When process operates in out-of-control states, the out-of-control transition probability matrix $\tilde{P}$ can be calculated in the same manner. The generic element $\tilde{p}_{j, k}$ is obtained:

$$
\begin{aligned}
& \tilde{p}_{j, k}=G\left(S_{j}+\phi_{h u}^{-1}\left(S_{k}-S_{j}+d / 2\right) \mid n-1\right)-G\left(S_{j}+\phi_{h u}^{-1}\left(S_{k}-S_{j}-d / 2\right) \mid n-1\right), \\
& \tilde{p}_{j, m+1}=G\left(\phi_{h u}^{-1}\left(L C L-S_{j}\right)+S_{j}\right)+1-G\left(\phi_{h u}^{-1}\left(U C L-S_{j}\right)+S_{j}\right) .
\end{aligned}
$$

The $l$-step transition probability matrix $P^{l}$ is the result of multiplication of $P$ by itself $l$ times. The matrix $P^{l}$ can be rewritten in partitioned form:

$$
P^{l}=\left[\begin{array}{cc}
A_{(2 m+1,2 m+1)}^{l} & W_{(2 m+1,2 m+1)}^{l} B_{(2 m+1,1)} \\
0_{(1,2 m+1)} & 1
\end{array}\right],
$$

where the matrix $W^{l}$ is defined as

$$
W^{l}=\sum_{i=0}^{l-1} A^{i} .
$$

The in-control TARL denoted by $\mathrm{TARL}_{0}$ can be calculated:

$$
\operatorname{TARL}_{0}=\underbrace{[0 \cdots 1 \cdots 0]}_{2 m+1} W^{I s+1} \underbrace{[1 \cdots 1 \cdots 1]}_{2 m+1} .
$$

The out-of-control TARL denoted by $\mathrm{TARL}_{1}$ can be calculated in the same manner by replacing $\tilde{P}$ to $P$ in Equation (15).

Another useful performance measure is $q(I s, \delta, \tau)$, which is defined as the probability of getting a signal within $I s$ inspections. When the process is in-control, $q(I s, 0,1)$ means that the probability that the AEWMA $t$ control chart signals a false alarm within Is inspections.

$$
\begin{aligned}
q(I s, 0,1) & =p_{0, m+1}^{I s} \\
& =\underbrace{[0 \cdots 1 \cdots 0]}_{2 m+1} W_{(2 m+1,2 m+1)}^{I s} B_{(2 m+1,1)},
\end{aligned}
$$

where $p_{0, m+1}^{I s}$ is the in-control transition probability from the transient state 0 to absorbing state $m+1$ of the Markov chain after $I s$ steps. When the process is out-of-control, $q(I s, \delta, \tau)$ represents the probability that AEWMA $t$ control chart signals a true alarm within Is samples. In the same manner, the value of $q(I s, \delta, \tau)$ can be calculated as the manner of equation (18).

The statistical design of AEWMA $t$ control chart is more complex than EWMA $t$ control chart. The out-of-control TARL at two mean shift sizes $\delta_{1}, \delta_{2}\left(\delta_{1}<\delta_{2}\right)$ have to be minimized subject to the in-control TARL equals to Is.

$$
\begin{array}{ll}
\min _{\theta} & \operatorname{TARL}_{1}\left(\delta_{1}, \theta\right) \quad \& \quad \min _{\theta} \operatorname{TARL}_{1}\left(\delta_{2}, \theta\right) \\
\text { s.t. } & \operatorname{TARL}_{0}(0, \theta)=I s
\end{array}
$$

where $\theta=(h, \lambda, \gamma)$ is the parameter set of AEWMA $t$ chart. 


\section{The Optimal Procedure of AEWMA $t$ Control Chart}

The statistical design of an AEWMA $t$ control chart is a 3-dimensional optimization problem, the parameter $h, \lambda$ and $\gamma$ are computed to satisfy equation (19). [16, 20] have investigated the effect of $\lambda$ and $\gamma$ alone on the chart performance. The joint effect of $\lambda$ and $\gamma$ on the run length performance of AEWMA $t$ control chart have been investigated by [18, 21]. However, the joint effect of $\lambda$ and $\gamma$ on the TARL performance for short production run has never been investigated. To better understand the overall effect, figure 1 shows contour plots of the outof-control TARL values of AEWMA $t$ chart as a function of $\lambda$ and $\gamma$ for $n=10 I s=10,30$, $\delta=0.5,2.0$ when zero-state in-control TARL is $I s$.

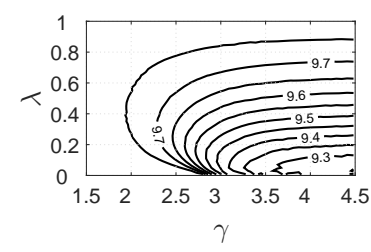

(a) $\mathrm{IS}=10 \quad \delta=0.5$

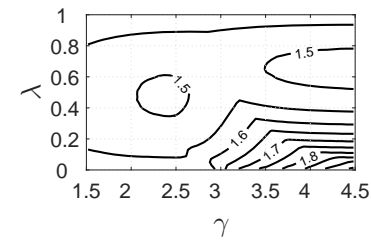

(c) $\quad \mathrm{Is}=10 \quad \delta=2.0$

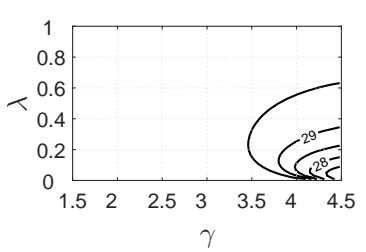

(b) $\quad \mathrm{Is}=30 \quad \delta=0.5$

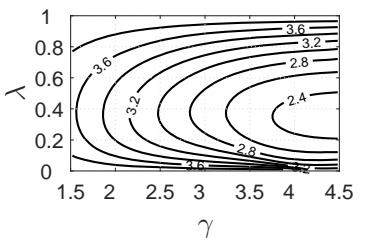

(d) $\quad$ Is $=30 \quad \delta=2.0$

Figure 1 Contour plots of TARL for different $I s$ and $\delta$

From Figures 1(a), 1(b) and 1(d), when detecting a small increase $(\delta=0.5)$ in the process mean, the TARL decreases as $\lambda$ decreases or $\gamma$ increases. From Figure 1(b), the TARL values are almost unchanged for $\gamma<3.5$. However, contour plots become more complex in figure $\mathbf{1}(\mathrm{c})$. The trend of TARL changing with $\lambda$ and $\gamma$ is no more obvious.

Two optimization procedures have been proposed by $[16,18]$, respectively. However, the procedure proposed by [18] suggests specifying an value of $\lambda$ to give the minimum value of ARL of an EWMA chart at small shift size $\delta_{1}$, firstly. Then, the optimal $\gamma$ value having the minimum ARL at $\delta_{2}$ is searched to satisfy the conditions that the percentage increase in the out-of-control ARL at $\delta_{1}$ is no more than a small positive number $\alpha$. However, Capizzi and Masarotto ${ }^{[16]}$ have suggested to find the optimal solution $\theta_{1}=(h, \lambda, \gamma)$ having the minimum $\operatorname{TARL}_{1}\left(\delta_{2}, \theta_{1}\right)$, firstly. Then, the optimal parameter $\theta_{2}=(h, \lambda, \gamma)$ having minimum $\operatorname{TARL}_{1}\left(\delta_{1}, \theta_{2}\right)$ is selected. The parameter $\theta_{2}=(h, \lambda, \gamma)$ has to be subject to the condition that the percentage increases of $\operatorname{TARL}_{1}\left(\delta_{2}, \theta_{1}\right)$ is at most of a small positive number $\alpha$. The procedure proposed by Capizzi and Masarotto ${ }^{[16]}$ is more complex than the procedure produced by Shu ${ }^{[18]}$, but the solutions of the method proposed by Capizzi and Masarotto ${ }^{[16]}$ are more efficient than that of Shu ${ }^{[18]}$.

Based on the analysis of joint effect of $\lambda$ and $\gamma$, a new two-stage optimization algorithm for short run AEWMA $t$ control chart is proposed. Details of the two-stage design procedure are summarized below: 
1) Specify the value of sample size $n$, the interval $\left[\lambda_{1}, \lambda_{2}\right]$ of $\lambda$ and the interval $\left[\gamma_{1}, \gamma_{2}\right]$ of $\gamma$; choose $n_{1}$ values of $\lambda$ from the interval of $\left[\lambda_{1}, \lambda_{2}\right]$; choose $n_{2}$ value of $\gamma$ from the interval of $\left[\gamma_{1}, \gamma_{2}\right]$; calculate the control limits $h$ for $n_{1} \times n_{2}$ combinations of $(\lambda, \gamma)$ subject to $\mathrm{TARL}_{0}=I s$.

2) Based on the solution $\theta=(h, \lambda, \gamma)$ of Step 1), calculate the TARL values of AEWMA control chart for different shift sizes $\delta_{1}, \delta_{2}\left(\delta_{1}<\delta_{2}\right)$; find the optimum solution $\theta=(h, \lambda, \gamma)$ having the minimum out-of-control TARL at $\delta_{1}, \delta_{2}$, which aredenoted as $\operatorname{TARL}_{1}\left(\delta_{1}, \theta_{1}^{*}\right)$ and $\operatorname{TARL}_{1}\left(\delta_{2}, \theta_{2}^{*}\right)$, respectively.

3) Choose two small positive constants $\alpha_{1}, \alpha_{2}$ (e.g. $\alpha_{1}=0.005, \alpha_{2}=0.05$ ); find the parameter $\theta$ subject to the following condition:

$$
\begin{aligned}
& \operatorname{TARL}_{1}\left(\delta_{1}, \theta\right)<\left(1+\alpha_{1}\right) \operatorname{TARL}_{1}\left(\delta_{1}, \theta_{1}^{*}\right), \\
& \operatorname{TARL}_{1}\left(\delta_{2}, \theta\right)<\left(1+\alpha_{2}\right) \operatorname{TARL}_{1}\left(\delta_{2}, \theta_{2}^{*}\right) .
\end{aligned}
$$

4) If non of the $\theta$ is found, increase the parameter $\alpha_{1}, \alpha_{2}$, and repeat Step 3) until the $\theta$ is found.

5) If only one $\theta$ is found, this is the final solution that can get the out-of-control TARL nearly minimum; otherwise, two or more $\theta$ (i.e., $\left.\theta_{j}, i=1,2, \cdots\right)$ are found, subdivide the interval $\left[\delta_{1}, \delta_{2}\right]$ into $l$ parts, calculate the TARL values of these shift sizes (i.e., $\delta_{i}, i=1,2, \cdots, l$ ) for each $\theta$; find the $\theta$ subject to the condition:

$$
\min _{\theta} \sum_{i=1}^{l} \operatorname{TARL}_{1}\left(\delta_{i}, \theta_{k}\right)
$$

The ranges of $\lambda$ and $\gamma$ is another important problem. Shu ${ }^{[18]}$ has suggested that $\lambda$ falls into the range $[0.1,0.4]$, and the value of $\gamma$ falls into the range $\left[1.5 \sigma_{T}, 3 \sigma_{T}\right]$. However, the $\lambda$ and $\gamma$ getted by Capizzi and Masarotto[16] have exceeded the ranges proposed by Shu ${ }^{[18]}$. From figure $\mathbf{1}(\mathrm{b})$ and $\mathbf{1}(\mathrm{d})$, it is declared that the up-limit of $\gamma$ should be greater than $3 \delta_{T}$. In this paper, the parameters $\lambda$ and $\gamma$ are within the range $(0,1)$ and $(0,10)$, respectively.

\section{Numerical Simulations}

To illustrate the performance of AEWMA $t$ chart, both the perfect and imperfect initial setup conditions are discussed. The performances of AEWMA $t$ chart are compared with the EWMA $t$ chart optimized at the shift sizes $\delta=0.5$ and $\delta=2.0$.

\subsection{Perfect Setup Condition}

Based on the optimal algorithm proposed in this paper, the optimal values of $\theta$ of AEWMA $t$ chart are calculated for the conditions: $n=5,10,25,50 ; I s=10,30, \tau=1$ and $\Delta=0$. The optimal solutions of $\theta$ are shown in Table 1 .

From Table 1, the values of $h$ and $\lambda$ increase with $n$, and the values of $\gamma$ decrease as $n$ increases. This trend is due to the large number of sample size $n$ would get the chart close to the condition that the process parameters are known previously. The EWMA $t$ control chart are optimized at $\delta=0.5,2.0$, and the optimal couples of $(h, \lambda)$ are shown in Table 2. From Table 2 , the values of $h$ increase with $n$, and the values of $\lambda$ increases with $n$ and $\delta$. This trend can also be found in [10]. 
Table 1 The optimal solutions of $\theta=(h, \lambda, \gamma)$

\begin{tabular}{cccccccc}
\hline & \multicolumn{3}{c}{$I s=10$} & & \multicolumn{3}{c}{$I s=30$} \\
\cline { 2 - 5 } \cline { 6 - 8 } & $h$ & $\lambda$ & $\gamma$ & & $h$ & $\lambda$ & $\gamma$ \\
\hline$n=5$ & 0.1682 & 0.03 & 7.90 & & 0.5390 & 0.05 & 9.95 \\
$n=10$ & 0.7608 & 0.21 & 5.50 & & 0.9679 & 0.16 & 9.85 \\
$n=25$ & 1.5895 & 0.60 & 5.10 & & 1.6460 & 0.41 & 6.40 \\
$n=50$ & 1.8610 & 0.51 & 1.15 & & 2.0800 & 0.52 & 2.50 \\
\hline
\end{tabular}

Table 2 The optimal solutions of $\theta=(h, \lambda, \gamma)$

\begin{tabular}{|c|c|c|c|c|c|c|c|c|}
\hline & \multicolumn{4}{|c|}{$\delta=0.5$} & \multicolumn{4}{|c|}{$\delta=2.0$} \\
\hline & \multicolumn{2}{|c|}{$I s=10$} & \multicolumn{2}{|c|}{$I s=30$} & \multicolumn{2}{|c|}{$I s=10$} & \multicolumn{2}{|c|}{$I s=30$} \\
\hline & $h$ & $\lambda$ & $h$ & $\lambda$ & $h$ & $\lambda$ & $h$ & $\lambda$ \\
\hline$n=5$ & 0.226 & 0.041 & 0.480 & 0.044 & 2.461 & 0.634 & 1.968 & 0.254 \\
\hline$n=10$ & 0.771 & 0.213 & 0.956 & 0.157 & 2.842 & 0.999 & 3.863 & 0.911 \\
\hline$n=25$ & 1.590 & 0.600 & 1.633 & 0.405 & 2.507 & 0.999 & 3.422 & 0.998 \\
\hline$n=50$ & 2.154 & 0.886 & 2.480 & 0.747 & 2.267 & 0.935 & 2.618 & 0.796 \\
\hline
\end{tabular}

Tables $3 \sim 6$ show the $\operatorname{TARL}_{1}(\delta, \theta)=\mathrm{TARL}_{1}$ and the $q(I s, \delta, \tau)$ of two investigated $t$-charts for $I s=10,30, \mathrm{TARL}_{0}=I s$. The optimal values of $\mathrm{TARL}_{1}$ and $q(I s, \delta, \tau)$ are indicated in bold in Tables $3 \sim 6$. Each value of $\tau$ corresponds to two rows of EWMA $t$ control chart optimized at $\delta=0.5$ and $\delta=2.0$, respectively. For example, in Table 3, when the shift size $\delta=0.5$, $\tau=1: \mathrm{TARL}_{1}=5.22$ for EWMA $t$ chart optimized at $\delta=0.5, \mathrm{TARL}_{1}=7.31$ for EWMA $t$ chart optimized at $\delta=2.0$. From Tables $3 \sim 6$, large sample sizes (i.e., $n>=25$ ) are welcomed to improve the statistical properties of $t$ charts. The non-central parameter $\psi$ decreases as $\tau$ increases leads to the sensitivity decrease of $t$ chart.

Table 3 TARL values of EWMA and AEWMA $t$ control chart for $\Delta=0, I s=10$

\begin{tabular}{|c|c|c|c|c|c|c|c|c|c|c|c|}
\hline \multirow[b]{3}{*}{$n$} & \multirow[b]{3}{*}{$\tau$} & \multicolumn{5}{|c|}{ EWMA } & \multicolumn{5}{|c|}{ AEWMA } \\
\hline & & \multicolumn{5}{|c|}{$\delta$} & \multicolumn{5}{|c|}{$\delta$} \\
\hline & & 0.00 & 0.50 & 1.00 & 1.50 & 2.00 & 0.00 & 0.50 & 1.00 & 1.50 & 2.00 \\
\hline \multirow{6}{*}{5} & \multirow{2}{*}{1.00} & 10.00 & 5.22 & 2.77 & 2.00 & 1.64 & \multirow{2}{*}{10.00} & \multirow{2}{*}{5.21} & \multirow{2}{*}{2.79} & \multirow{2}{*}{2.02} & \multirow{2}{*}{1.66} \\
\hline & & 10.00 & 7.31 & 3.22 & 1.81 & 1.33 & & & & & \\
\hline & \multirow{2}{*}{1.50} & 10.00 & 7.13 & 3.99 & 2.77 & 2.19 & \multirow{2}{*}{10.00} & \multirow{2}{*}{7.10} & \multirow{2}{*}{4.00} & \multirow{2}{*}{2.79} & \multirow{2}{*}{2.21} \\
\hline & & 10.00 & 8.74 & 5.69 & 3.22 & 2.10 & & & & & \\
\hline & \multirow{2}{*}{2.00} & 10.00 & 8.20 & 5.22 & 3.58 & 2.77 & \multirow{2}{*}{10.00} & \multirow{2}{*}{8.18} & \multirow{2}{*}{5.21} & \multirow{2}{*}{3.59} & \multirow{2}{*}{2.79} \\
\hline & & 10.00 & 9.29 & 7.31 & 4.93 & 3.22 & & & & & \\
\hline \multirow{6}{*}{10} & \multirow{2}{*}{1.00} & 10.00 & 3.29 & 1.66 & 1.17 & 1.02 & \multirow{2}{*}{10.00} & \multirow{2}{*}{3.28} & \multirow{2}{*}{1.67} & \multirow{2}{*}{1.18} & \multirow{2}{*}{1.02} \\
\hline & & 10.00 & 5.30 & 1.58 & 1.05 & 1.00 & & & & & \\
\hline & \multirow{2}{*}{1.50} & 10.00 & 5.16 & 2.42 & 1.66 & 1.30 & \multirow{2}{*}{10.00} & \multirow{2}{*}{5.15} & 2.42 & 1.67 & 1.30 \\
\hline & & 10.00 & 7.64 & 3.33 & 1.58 & 1.13 & & & 2.42 & 1.06 & 1.30 \\
\hline & 2.00 & 10.00 & 6.68 & 3.29 & 2.16 & 1.66 & 10.00 & 6.66 & 3.28 & 2.16 & 1.67 \\
\hline & & 10.00 & 8.64 & 5.30 & 2.66 & 1.58 & & & & & \\
\hline
\end{tabular}




\begin{tabular}{|c|c|c|c|c|c|c|c|c|c|c|c|}
\hline \multirow{6}{*}{25} & \multirow{2}{*}{1.00} & 10.00 & 1.76 & 1.01 & 1.00 & 1.00 & \multirow{2}{*}{10.00} & \multirow{2}{*}{1.76} & \multirow{2}{*}{1.01} & \multirow{2}{*}{1.00} & \multirow{2}{*}{1.00} \\
\hline & & 10.00 & 1.97 & 1.01 & 1.00 & 1.00 & & & & & \\
\hline & \multirow{2}{*}{1.50} & 10.00 & 3.14 & 1.27 & 1.01 & 1.00 & \multirow{2}{*}{10.00} & \multirow{2}{*}{3.14} & \multirow{2}{*}{1.27} & \multirow{2}{*}{1.01} & \multirow{2}{*}{1.00} \\
\hline & & 10.00 & 4.23 & 1.27 & 1.01 & 1.00 & & & & & \\
\hline & \multirow{2}{*}{2.00} & 10.00 & 4.78 & 1.76 & 1.15 & 1.01 & \multirow{2}{*}{10.00} & \multirow{2}{*}{4.78} & \multirow{2}{*}{1.76} & \multirow{2}{*}{1.15} & \multirow{2}{*}{1.01} \\
\hline & & 10.00 & 6.21 & 1.97 & 1.13 & 1.01 & & & & & \\
\hline \multirow{6}{*}{50} & \multirow{2}{*}{1.00} & 10.00 & 1.15 & 1.00 & 1.00 & 1.00 & \multirow{2}{*}{10.00} & \multirow{2}{*}{1.15} & \multirow{2}{*}{1.00} & \multirow{2}{*}{1.00} & \multirow{2}{*}{1.00} \\
\hline & & 10.00 & 1.16 & 1.00 & 1.00 & 1.00 & & & & & \\
\hline & \multirow{2}{*}{1.50} & 10.00 & 1.96 & 1.01 & 1.00 & 1.00 & \multirow{2}{*}{10.00} & \multirow{2}{*}{1.97} & \multirow{2}{*}{1.01} & \multirow{2}{*}{1.00} & \multirow{2}{*}{1.00} \\
\hline & & 10.00 & 2.00 & 1.01 & 1.00 & 1.00 & & & & & \\
\hline & \multirow{2}{*}{2.00} & 10.00 & 3.28 & 1.15 & 1.00 & 1.00 & \multirow{2}{*}{10.00} & \multirow{2}{*}{3.35} & \multirow{2}{*}{1.15} & \multirow{2}{*}{1.00} & 1.00 \\
\hline & & 10.00 & 3.42 & 1.16 & 1.00 & 1.00 & & & & & \\
\hline
\end{tabular}

Table 4 TARL values of EWMA and AEWMA $t$ control chart for $\Delta=0, I s=30$

\begin{tabular}{|c|c|c|c|c|c|c|c|c|c|c|c|}
\hline \multirow[b]{3}{*}{$n$} & \multirow[b]{3}{*}{$\tau$} & \multicolumn{5}{|c|}{ EWMA } & \multicolumn{5}{|c|}{ AEWMA } \\
\hline & & \multicolumn{5}{|c|}{$\delta$} & \multicolumn{5}{|c|}{$\delta$} \\
\hline & & 0.00 & 0.50 & 1.00 & 1.50 & 2.00 & 0.00 & 0.50 & 1.00 & 1.50 & 2.00 \\
\hline \multirow{6}{*}{5} & \multirow{2}{*}{1.00} & 30.00 & 10.46 & 4.98 & 3.40 & 2.66 & \multirow{2}{*}{30.00} & \multirow{2}{*}{10.61} & \multirow{2}{*}{4.97} & \multirow{2}{*}{3.38} & \multirow{2}{*}{2.63} \\
\hline & & 30.00 & 17.76 & 5.26 & 3.02 & 2.22 & & & & & \\
\hline & \multirow{2}{*}{1.50} & 30.00 & 16.61 & 7.58 & 4.98 & 3.78 & \multirow{2}{*}{30.00} & \multirow{2}{*}{16.99} & \multirow{2}{*}{7.63} & \multirow{2}{*}{4.97} & \multirow{2}{*}{3.76} \\
\hline & & 30.00 & 24.98 & 11.00 & 5.26 & 3.49 & & & & & \\
\hline & \multirow{2}{*}{2.00} & 30.00 & 21.45 & 10.46 & 6.68 & 4.98 & \multirow{2}{*}{30.00} & \multirow{2}{*}{21.88} & \multirow{2}{*}{10.61} & \multirow{2}{*}{6.71} & \multirow{2}{*}{4.97} \\
\hline & & 30.00 & 27.44 & 17.76 & 8.76 & 5.26 & & & & & \\
\hline & 100 & 30.00 & 5.54 & 2.52 & 1.79 & 1.40 & 3000 & 554 & 251 & 178 & 139 \\
\hline & 1.00 & 30.00 & 18.29 & 3.46 & 1.43 & 1.06 & 30.00 & 0.04 & 2.01 & 1.10 & 1.05 \\
\hline \multirow[t]{6}{*}{10} & \multirow{2}{*}{1.50} & 30.00 & 9.96 & 3.88 & 2.52 & 1.95 & \multirow{2}{*}{30.00} & \multirow{2}{*}{9.99} & 387 & 251 & 195 \\
\hline & & 30.00 & 25.26 & 10.66 & 3.46 & 1.76 & & & & & \\
\hline & 200 & 30.00 & 15.25 & 5.54 & 3.40 & 2.52 & 3000 & 1530 & 5.54 & 3.38 & 2.51 \\
\hline & & 30.00 & 27.53 & 18.29 & 7.81 & 3.46 & & & & & \\
\hline & 100 & 30.00 & 2.66 & 1.19 & 1.00 & 1.00 & 3000 & 266 & 110 & 00 & OR \\
\hline & 1.00 & 30.00 & 4.70 & 1.08 & 1.00 & 1.00 & 50.00 & 2.00 & 1.19 & 1.00 & 1.00 \\
\hline 25 & 150 & 30.00 & 5.15 & 1.86 & 1.19 & 1.01 & 30.00 & 5.77 & 1.85 & 1.19 & 01 \\
\hline & & 30.00 & 14.13 & 2.08 & 1.08 & 1.00 & & & & & \\
\hline & 200 & 30.00 & 9.20 & 2.66 & 1.63 & 1.19 & 3000 & 9.26 & 2.66 & 163 & 119 \\
\hline & & 30.00 & 21.18 & 4.70 & 1.60 & 1.08 & & & & & \\
\hline & 100 & 30.00 & 1.51 & 1.00 & 1.00 & 1.00 & 30.00 & 1.49 & 1.00 & 1.00 & 1.00 \\
\hline & & 30.00 & 1.52 & 1.00 & 1.00 & 1.00 & & & & & \\
\hline 50 & 150 & 30.00 & 3.28 & 1.09 & 1.00 & 1.00 & 30 م 3 & 3.10 & 09 & 100 & 00 \\
\hline & 1.00 & 30.00 & 3.47 & 1.09 & 1.00 & 1.00 & 50.00 & 0.10 & 1.08 & 1.00 & 1.00 \\
\hline & 200 & 30.00 & 6.75 & 1.51 & 1.03 & 1.00 & 3000 & 604 & 149 & 103 & 100 \\
\hline & & 30.00 & 7.41 & 1.52 & 1.03 & 1.00 & 30.00 & 0.04 & 1.49 & 1.03 & \\
\hline
\end{tabular}


Table $5 q$ values of EWMA and AEWMA $t$ control chart for $\Delta=0, I s=10$

\begin{tabular}{|c|c|c|c|c|c|c|c|c|c|c|c|}
\hline \multirow[b]{3}{*}{$n$} & \multirow[b]{3}{*}{$\tau$} & \multicolumn{5}{|c|}{ EWMA } & \multicolumn{5}{|c|}{ AEWMA } \\
\hline & & \multicolumn{5}{|c|}{$\delta$} & \multicolumn{5}{|c|}{$\delta$} \\
\hline & & 0.00 & 0.50 & 1.00 & 1.50 & 2.00 & 0.00 & 0.50 & 1.00 & 1.50 & 2.00 \\
\hline \multirow{6}{*}{5} & \multirow{2}{*}{1.00} & 0.222 & 0.965 & 1.000 & 1.000 & 1.000 & \multirow{2}{*}{0.227} & \multirow{2}{*}{0.967} & \multirow{2}{*}{1.000} & \multirow{2}{*}{1.000} & \multirow{2}{*}{1.000} \\
\hline & & 0.178 & 0.617 & 0.987 & 1.000 & 1.000 & & & & & \\
\hline & 150 & 0.222 & 0.779 & 0.998 & 1.000 & 1.000 & \multirow{2}{*}{0.227} & \multirow{2}{*}{0.787} & \multirow{2}{*}{0.998} & \multirow{2}{*}{1.000} & \multirow{2}{*}{1.000} \\
\hline & 1.00 & 0.178 & 0.396 & 0.819 & 0.987 & 1.000 & & & & & \\
\hline & \multirow{2}{*}{2.00} & 0.222 & 0.605 & 0.965 & 1.000 & 1.000 & \multirow{2}{*}{0.227} & \multirow{2}{*}{0.614} & \multirow{2}{*}{0.967} & \multirow{2}{*}{1.000} & \multirow{2}{*}{1.000} \\
\hline & & 0.178 & 0.304 & 0.617 & 0.891 & 0.987 & & & & & \\
\hline \multirow{6}{*}{10} & \multirow{2}{*}{1.00} & 0.201 & 0.998 & 1.000 & 1.000 & 1.000 & \multirow{2}{*}{0.203} & \multirow{2}{*}{0.999} & \multirow{2}{*}{1.000} & 0 & 000 \\
\hline & & 0.177 & 0.828 & 1.000 & 1.000 & 1.000 & & & & & \\
\hline & 150 & 0.201 & 0.931 & 1.000 & 1.000 & 1.000 & ( 203 & 3 & 10 & 1000 & 10 \\
\hline & 1.00 & 0.177 & 0.547 & 0.969 & 1.000 & 1.000 & 0.200 & 0 & 1.0 & 1.000 & 1.0 \\
\hline & 2.00 & 0.201 & 0.779 & 0.998 & 1.000 & 1.000 & 203 & & & & 1.000 \\
\hline & 2.00 & 0.177 & 0.399 & 0.828 & 0.991 & 1.000 & 203 & & .99 & .000 & 1.000 \\
\hline & 1.00 & 0.182 & 1.000 & 1.000 & 1.000 & 1.000 & 0.182 & 1.000 & 1.000 & 1.000 & 1.000 \\
\hline & & 0.177 & 0.999 & 1.000 & 1.000 & 1.000 & & & & & \\
\hline 25 & 1.50 & 0.182 & 0.991 & 1.000 & 1.000 & 1.000 & 0.182 & 0.991 & 1.000 & 1.000 & 1.000 \\
\hline & & 0.177 & 0.918 & 1.000 & 1.000 & 1.000 & & & & & \\
\hline & 200 & 0.182 & 0.909 & 1.000 & 1.000 & 1.000 & 0182 & 0909 & 1000 & 1000 & 1000 \\
\hline & 2.00 & 0.177 & 0.732 & 0.999 & 1.000 & 1.000 & 0.102 & 0.808 & 1.000 & 1.000 & 1.000 \\
\hline & 1.00 & 0.177 & 1.000 & 1.000 & 1.000 & 1.000 & & & & & \\
\hline & 1.00 & 0.177 & 1.000 & 1.000 & 1.000 & 1.000 & 0.179 & .000 & 1.000 & .000 & .000 \\
\hline 50 & 1.50 & 0.177 & 1.000 & 1.000 & 1.000 & 1.000 & 179 & & & & \\
\hline & 1.00 & 0.177 & 0.999 & 1.000 & 1.000 & 1.000 & 0.189 & 1.000 & 1.000 & .000 & 1.0 \\
\hline & 2.00 & 0.177 & 0.976 & 1.000 & 1.000 & 1.000 & 0.179 & 0.975 & 1.000 & 1.000 & 1.000 \\
\hline & & 0.177 & 0.968 & 1.000 & 1.000 & 1.000 & & & & & \\
\hline
\end{tabular}

Table $6 q$ values of EWMA and AEWMA $t$ control chart for $\Delta=0, I s=30$

\begin{tabular}{|c|c|c|c|c|c|c|c|c|c|c|c|}
\hline \multirow[b]{3}{*}{$n$} & \multirow[b]{3}{*}{$\tau$} & \multicolumn{5}{|c|}{ EWMA } & \multicolumn{5}{|c|}{ AEWMA } \\
\hline & & \multicolumn{5}{|c|}{$\delta$} & \multicolumn{5}{|c|}{$\delta$} \\
\hline & & 0.00 & 0.50 & 1.00 & 1.50 & 2.00 & 0.00 & 0.50 & 1.00 & 1.50 & 2.00 \\
\hline \multirow{6}{*}{5} & \multirow{2}{*}{1.00} & 0.082 & 0.999 & 1.000 & 1.000 & 1.000 & \multirow{2}{*}{0.080} & \multirow{2}{*}{0.999} & \multirow{2}{*}{1.000} & \multirow{2}{*}{1.000} & \multirow{2}{*}{1.000} \\
\hline & & 0.065 & 0.779 & 1.000 & 1.000 & 1.000 & & & & & \\
\hline & \multirow{2}{*}{1.50} & 0.082 & 0.930 & 1.000 & 1.000 & 1.000 & \multirow{2}{*}{0.080} & \multirow{2}{*}{0.916} & \multirow{2}{*}{1.000} & \multirow{2}{*}{1.000} & \multirow{2}{*}{1.000} \\
\hline & & 0.065 & 0.400 & 0.973 & 1.000 & 1.000 & & & & & \\
\hline & \multirow{2}{*}{2.00} & 0.082 & 0.734 & 0.999 & 1.000 & 1.000 & \multirow{2}{*}{0.0800} & \multirow{2}{*}{0.7052} & \multirow{2}{*}{0.9991} & \multirow{2}{*}{1.0000} & \multirow{2}{*}{1.0000} \\
\hline & & 0.065 & 0.242 & 0.779 & 0.995 & 1.000 & & & & & \\
\hline
\end{tabular}




\begin{tabular}{|c|c|c|c|c|c|c|c|c|c|c|c|}
\hline \multirow{6}{*}{10} & \multirow{2}{*}{1.00} & 0.072 & 1.000 & 1.000 & 1.000 & 1.000 & \multirow{2}{*}{0.072} & \multirow{2}{*}{1.000} & \multirow{2}{*}{1.000} & \multirow{2}{*}{1.000} & \multirow{2}{*}{1.000} \\
\hline & & 0.064 & 0.695 & 1.000 & 1.000 & 1.000 & & & & & \\
\hline & \multirow{2}{*}{1.50} & 0.072 & 0.993 & 1.000 & 1.000 & 1.000 & \multirow{2}{*}{0.072} & \multirow{2}{*}{0.992} & \multirow{2}{*}{1.000} & \multirow{2}{*}{1.000} & \multirow{2}{*}{1.000} \\
\hline & & 0.064 & 0.348 & 0.941 & 1.000 & 1.000 & & & & & \\
\hline & \multirow{2}{*}{2.00} & 0.072 & 0.900 & 1.000 & 1.000 & 1.000 & \multirow{2}{*}{0.072} & \multirow{2}{*}{0.898} & \multirow{2}{*}{1.000} & \multirow{2}{*}{1.000} & \multirow{2}{*}{1.000} \\
\hline & & 0.064 & 0.216 & 0.695 & 0.984 & 1.000 & & & & & \\
\hline \multirow{6}{*}{25} & \multirow{2}{*}{1.00} & 0.067 & 1.000 & 1.000 & 1.000 & 1.000 & \multirow{2}{*}{0.067} & \multirow{2}{*}{1.000} & \multirow{2}{*}{1.000} & \multirow{2}{*}{1.000} & \multirow{2}{*}{1.000} \\
\hline & & 0.064 & 0.999 & 1.000 & 1.000 & 1.000 & & & & & \\
\hline & 1.50 & 0.067 & 1.000 & 1.000 & 1.000 & 1.000 & \multirow{2}{*}{0.067} & \multirow{2}{*}{1.000} & \multirow{2}{*}{1.0000} & \multirow{2}{*}{1.0000} & \multirow{2}{*}{1.0000} \\
\hline & & 0.064 & 0.847 & 1.000 & 1.000 & 1.000 & & & & & \\
\hline & \multirow{2}{*}{2.00} & 0.067 & 0.984 & 1.000 & 1.000 & 1.000 & 0067 & 0983 & 1000 & 1000 & 1000 \\
\hline & & 0.064 & 0.560 & 0.999 & 1.000 & 1.000 & & & & & \\
\hline & 100 & 0.064 & 1.000 & 1.000 & 1.000 & 1.000 & 0065 & 0 & 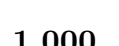 & 0 & 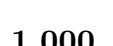 \\
\hline & 1.00 & 0.064 & 1.000 & 1.000 & 1.000 & 1.000 & 0.000 & 1.000 & 1.000 & 1.000 & 1.000 \\
\hline 50 & 150 & 0.064 & 1.000 & 1.000 & 1.000 & 1.000 & 0065 & 1000 & 0 & 000 & 1000 \\
\hline & 1.00 & 0.064 & 1.000 & 1.000 & 1.000 & 1.000 & 0.000 & 1.000 & 1.000 & 1.000 & 1.000 \\
\hline & 200 & 0.064 & 0.995 & 1.000 & 1.000 & 1.000 & 0065 & 0999 & 1000 & 1000 & 1000 \\
\hline & 2.00 & 0.064 & 0.990 & 1.000 & 1.000 & 1.000 & 0.000 & 0.999 & 1.000 & 1.000 & 1.000 \\
\hline
\end{tabular}

Obviously, from Tables 3 and 4 , for small size of $\delta$ (i.e., $\delta<1.0$ ), both EWMA $t$ chart optimized at $\delta=0.5$ and AEWMA $t$ chart perform better than EWMA $t$ chart optimized at $\delta=2.0$. However, when mean shift size gets large (i.e., $\delta>1.5$ ), the EWMA $t$ chart optimized at $\delta=2.0$ perform better than EWMA $t$ chart optimized at $\delta=0.5$. That is to say, the TARL of AEWMA $t$ chart is always optimal or close to optimal. For small sample size $n \leq 10$ and large mean shift $\delta<1$ : The ratio of AEWMA $t$ chart gets the optimal TARL $\mathrm{T}_{1}$ is $11 / 12$, and the maximum deviation from the optimal $\mathrm{TARL}_{1}$ is $0.28 \%((3.59-3.58) / 3.58)$. For small sample size $n \leq 10$ and large mean shift $\delta>1.5$ : The ratio of AEWMA $t$ chart gets the optimal $1 / 12$, and the maximum deviation from the optimal $\mathrm{TARL}_{1}$ is $24.8 \%((1.66-1.33) / 1.33)$. However, when sample size $n$ is large $n \geq 25$ : The ratio of AEWMA $t$ chart gets the optimal TARL $_{1}$ is $10 / 12$, $11 / 12$; and the maximum deviation from the optimal $\mathrm{TARL}_{1}$ is $1.77 \%((1.15-1.13) / 1.13)$, $0.96 \%((1.05-1.04) / 1.04)$ for $\delta<1.0$ and $\delta>1.5$ respectively. When the scheduled inspection number increases $(I s=30)$ in Table 4 , the AEWMA $t$ chart show the same trend as in Table 4. But, the ratio of optimal TARL 1 of AEWMA $t$ chart is smaller than that in Table 3.

Tables 5 and 6 show the values of $q(I s, \delta, \tau)$. When the process is in control, the EWMA $t$ chart always gets the minimum probabilities of signal a false alarm within the $I s$ samples: $0.1766 \sim 0.1781$ for $I s=10$, and $0.0641 \sim 0.0654$ for $I s=30$. The probabilities of signal a false alarm within the $I s$ samples of AEWMA $t$ chart is: $0.1788 \sim 0.2268$ for $I s=10$, and $0.0649 \sim 0.0800$ for $I s=30$. However, when the sample size $n \geq 25$, the probabilities of AEWMA $t$ chart signals a false alarm within the $I s$ samples are close to the EWMA $t$ chart: $0.1788 \sim 0.1822$ for $I s=10$, and $0.0649 \sim 0.0670$ for $I s=30$. From Tables 5 and 6 , the probabilities of AEWMA $t$ chart to signal a true alarm are almost all optimal. In Table 5 , the ratio of AEWMA $t$ chart gets the optimal $q(I s, \delta, \tau)$ is $98.81 \% 83 / 84$ when $I s$ equals to 10 . The probabilities of AEWMA $t$ chart to signal a true alarm decrease as $I s$ increases. In Table 6 , 
the ratio of AEWMA $t$ chart gets the optimal $q(I s, \delta, \tau)$ is $80.95 \% 34 / 42$ when $n<25$. When $n \geq 25$, the ratio of AEWMA $t$ chart gets the optimal $q(I s, \delta, \tau)$ is $97.62 \% 41 / 42$.

To sum up, the AEWMA $t$ chart always performs best or or near to best. The large sample size or small number of schedule inspection will improve the AEWMA $t$ chart performance. In most cases, the AEWMA $t$ chart is more powerful than the traditional EWMA $t$ chart. In practice, applying one AEWMA $t$ chart is more convenience than applying several EWMA $t$ chart optimized at different mean shifts simultaneously.

\subsection{Imperfect Setup Condition}

When the process begins with an initial setup error $(\Delta \neq 0)$, the distribution function of $T$ is same as the perfect setup condition of $\delta=\Delta$. Namely, the initial setup error changes the value of $\delta$. However, the mean shift size $\delta=-\Delta$ should be specifically noted. From Table 1 , we know that $\psi$ equals to zero with the form of $(\delta+\Delta) \sqrt{n}$ and $((\delta+\Delta) / \tau) \sqrt{n}$ for $\delta=-\Delta$. Mathematically speaking, the imperfect setup process is in-control for $\delta=-\Delta$. The TARL of AEWMA chart for $n=10$ is shown in Figure 2 .

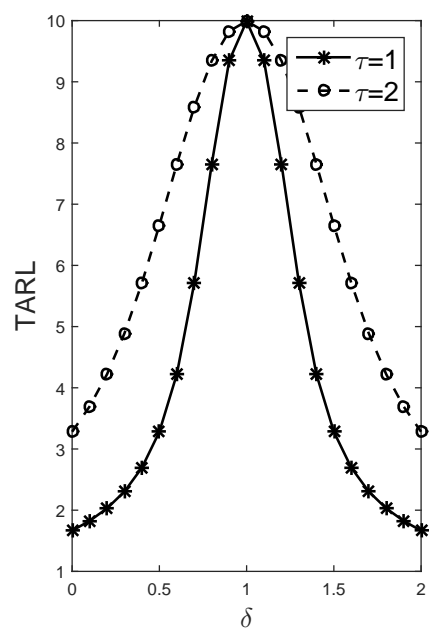

(a) $\Delta=-1 \quad \mathrm{I}_{\mathrm{s}}=10$

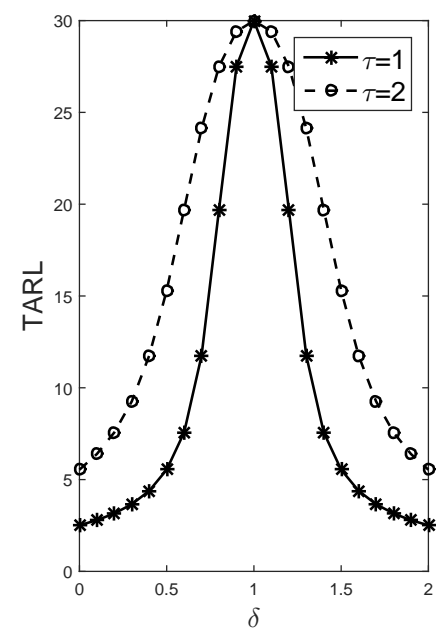

(b) $\Delta=-1 \quad \mathrm{I}_{\mathrm{s}}=30$

Figure 2 TARL values of AEWMA $t$ chart with the imperfect initial setup condition

The out-of-control $T A R L$ and $q(I s, \delta, \tau)$ are calculated for $n=5,10,25,50 ; I s=10 ; \tau=1$ and $\Delta=-1$. The results are shown in Table 7 and Table 8 . Obviously, each row is symmetrical about $\delta=1$, due to $\delta+\Delta=0$. Other properties are same as the perfect-setup condition. From Table 7, the ratio of AEWMA chart gets the optimal TARL is 78.57\%(66/84). Almost all the non-optimal TARL are very close to the optimal values (the deviations are less than 0.0072 ), except $n=5, \delta=0.75,1.25$ (the deviations are 5.6\%(0.18/3.17), 34.43\%(1.57/4.56), $31.4 \%(1.84 / 5.56)$ for $\tau=1,1.5,2.0$, respectively). From Table 8, the ratio of AEWMA chart gets the optimal $q(I s, \delta, \tau)$ is $88.10 \%(74 / 84)$. 
Table 7 TARL values of EWMA and AEWMA $t$ control chart for $\Delta=-1, I s=10$

\begin{tabular}{|c|c|c|c|c|c|c|c|c|c|c|c|}
\hline \multirow[b]{3}{*}{$n$} & \multirow[b]{3}{*}{$\tau$} & \multicolumn{5}{|c|}{ EWMA } & \multicolumn{5}{|c|}{ AEWMA } \\
\hline & & \multicolumn{5}{|c|}{$\delta$} & \multicolumn{5}{|c|}{$\delta$} \\
\hline & & 0.00 & 0.50 & 1.00 & 1.50 & 2.00 & 0.00 & 0.50 & 1.00 & 1.50 & 2.00 \\
\hline \multirow{6}{*}{5} & \multirow{2}{*}{1.00} & 2.77 & 5.22 & 10.00 & 5.22 & 2.77 & \multirow{2}{*}{2.79} & \multirow{2}{*}{5.21} & \multirow{2}{*}{10.00} & \multirow{2}{*}{5.21} & \multirow{2}{*}{2.79} \\
\hline & & 3.22 & 7.31 & 10.00 & 7.31 & 3.22 & & & & & \\
\hline & \multirow{2}{*}{1.50} & 3.99 & 7.13 & 10.00 & 7.13 & 3.99 & \multirow{2}{*}{4.00} & \multirow{2}{*}{7.10} & \multirow{2}{*}{10.00} & \multirow{2}{*}{7.10} & \multirow{2}{*}{4.00} \\
\hline & & 5.69 & 8.74 & 10.00 & 8.74 & 5.69 & & & & & \\
\hline & \multirow{2}{*}{2.00} & 5.22 & 8.20 & 10.00 & 8.20 & 5.22 & \multirow{2}{*}{5.21} & \multirow{2}{*}{8.18} & \multirow{2}{*}{10.00} & \multirow{2}{*}{8.18} & \multirow{2}{*}{5.21} \\
\hline & & 7.31 & 9.29 & 10.00 & 9.29 & 7.31 & & & & & \\
\hline \multirow{6}{*}{10} & \multirow{2}{*}{1.00} & 1.66 & 3.29 & 10.00 & 3.29 & 1.66 & \multirow{2}{*}{1.67} & \multirow{2}{*}{3.28} & 0 & 28 & 67 \\
\hline & & 1.58 & 5.30 & 10.00 & 5.30 & 1.58 & & & 10.00 & .20 & 1.01 \\
\hline & 1.50 & 2.42 & 5.16 & 10.00 & 5.16 & 2.42 & 42 & 5.15 & 0. & 5 & 2 \\
\hline & 1.50 & 3.33 & 7.64 & 10.00 & 7.64 & 3.33 & 42 & 5 & 0.00 & 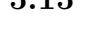 & .42 \\
\hline & 2.00 & 3.29 & 6.68 & 10.00 & 6.68 & 3.29 & 3.28 & 6.66 & 10.00 & 6.66 & 3.28 \\
\hline & & 5.30 & 8.64 & 10.00 & 8.64 & 5.30 & & & & & \\
\hline & 100 & 1.01 & 1.76 & 10.00 & 1.76 & 1.01 & 101 & 176 & 1000 & 176 & 101 \\
\hline & 1.00 & 1.01 & 1.97 & 10.00 & 1.97 & 1.01 & 1.01 & 1.00 & 10.00 & 1.00 & 1.01 \\
\hline 25 & 1.50 & 1.27 & 3.14 & 10.00 & 3.14 & 1.27 & 1.27 & 4 & 10 & 14 & 1.27 \\
\hline & & 1.27 & 4.23 & 10.00 & 4.23 & 1.27 & 1.21 & & 10.00 & 0.14 & 102 \\
\hline & 2.00 & 1.76 & 4.78 & 10.00 & 4.78 & 1.76 & 76 & 4.78 & 10.00 & 4.78 & 1.76 \\
\hline & & 1.97 & 6.21 & 10.00 & 6.21 & 1.97 & & & & & 1.10 \\
\hline & 1.00 & 1.00 & 1.15 & 10.00 & 1.15 & 1.00 & 1.00 & 1.15 & 10.00 & 1.15 & 1.00 \\
\hline & & 1.01 & 1.84 & 10.00 & 1.84 & 1.01 & & & & & \\
\hline 50 & 1.50 & 1.01 & 1.96 & 10.00 & 1.96 & 1.01 & 1.01 & 1.97 & 10.00 & 1.97 & 1.01 \\
\hline & & 1.41 & 2.48 & 10.00 & 2.48 & 1.41 & & & & & \\
\hline & 2.00 & 1.15 & 3.17 & 10.00 & 3.17 & 1.15 & 1.15 & 3.35 & 10.00 & 3.35 & 1.15 \\
\hline & & 1.84 & 3.17 & 10.00 & 3.28 & 1.84 & & & & & \\
\hline
\end{tabular}

Table $8 q$ values of EWMA and AEWMA $t$ control chart for $\Delta=-1, I s=10$

\begin{tabular}{|c|c|c|c|c|c|c|c|c|c|c|c|}
\hline \multirow[b]{3}{*}{$n$} & \multirow[b]{3}{*}{$\tau$} & \multicolumn{5}{|c|}{ EWMA } & \multicolumn{5}{|c|}{ AEWMA } \\
\hline & & \multicolumn{5}{|c|}{$\delta$} & \multicolumn{5}{|c|}{$\delta$} \\
\hline & & 0.00 & 0.50 & 1.00 & 1.50 & 2.00 & 0.00 & 0.50 & 1.00 & 1.50 & 2.00 \\
\hline & \multirow{2}{*}{1.00} & 1.000 & 0.965 & 0.222 & 0.965 & 1.000 & \multirow{2}{*}{1.000} & \multirow{2}{*}{0.967} & \multirow{2}{*}{0.227} & \multirow{2}{*}{0.967} & \multirow{2}{*}{1.000} \\
\hline & & 0.987 & 0.617 & 0.178 & 0.617 & 0.987 & & & & & \\
\hline \multirow[t]{4}{*}{5} & 1.50 & 0.998 & 0.779 & 0.222 & 0.779 & 0.998 & \multirow{2}{*}{0.998} & \multirow{2}{*}{0.787} & \multirow{2}{*}{0.227} & \multirow{2}{*}{0.787} & \multirow{2}{*}{0.998} \\
\hline & & 0.819 & 0.396 & 0.178 & 0.396 & 0.819 & & & & & \\
\hline & \multirow{2}{*}{2.00} & 0.965 & 0.605 & 0.222 & 0.605 & 0.965 & \multirow{2}{*}{0.967} & \multirow{2}{*}{0.614} & \multirow{2}{*}{0.227} & \multirow{2}{*}{0.614} & \multirow{2}{*}{0.967} \\
\hline & & 0.617 & 0.304 & 0.178 & 0.304 & 0.617 & & & & & \\
\hline
\end{tabular}




\begin{tabular}{|c|c|c|c|c|c|c|c|c|c|c|c|}
\hline \multirow{2}{*}{\multicolumn{2}{|c|}{1.00}} & 1.000 & 0.998 & 0.201 & 0.998 & 1.000 & \multirow{2}{*}{1.000} & \multirow{2}{*}{0.999} & \multirow{2}{*}{0.203} & \multirow{2}{*}{0.999} & \multirow[t]{2}{*}{1.000} \\
\hline & & 1.000 & 0.828 & 0.177 & 0.828 & 1.000 & & & & & \\
\hline \multirow[t]{4}{*}{10} & \multirow{2}{*}{1.50} & 1.000 & 0.931 & 0.201 & 0.931 & 1.000 & \multirow{2}{*}{1.000} & \multirow{2}{*}{0.933} & \multirow{2}{*}{0.203} & \multirow{2}{*}{0.933} & \multirow{2}{*}{1.000} \\
\hline & & 0.969 & 0.547 & 0.177 & 0.547 & 0.969 & & & & & \\
\hline & 2.00 & 0.998 & 0.779 & 0.201 & 0.779 & 0.998 & \multirow{2}{*}{0.999} & \multirow{2}{*}{0.782} & \multirow{2}{*}{0.203} & \multirow{2}{*}{0.782} & \multirow{2}{*}{0.999} \\
\hline & & 0.828 & 0.399 & 0.177 & 0.399 & 0.828 & & & & & \\
\hline \multirow{6}{*}{25} & \multirow{2}{*}{1.00} & 1.000 & 1.000 & 0.182 & 1.000 & 1.000 & \multirow{2}{*}{1.000} & \multirow{2}{*}{1.000} & \multirow{2}{*}{0.182} & \multirow{2}{*}{1.000} & \multirow{2}{*}{1.000} \\
\hline & & 1.000 & 0.999 & 0.177 & 0.999 & 1.000 & & & & & \\
\hline & 1.50 & 1.000 & 0.991 & 0.182 & 0.991 & 1.000 & \multirow{2}{*}{1.000} & \multirow{2}{*}{0.991} & \multirow{2}{*}{0.182} & \multirow{2}{*}{0.991} & \multirow{2}{*}{1.000} \\
\hline & 1.00 & 1.000 & 0.918 & 0.177 & 0.918 & 1.000 & & & & & \\
\hline & \multirow{2}{*}{2.00} & 1.000 & 0.909 & 0.182 & 0.909 & 1.000 & \multirow{2}{*}{1.000} & \multirow{2}{*}{0.909} & 0182 & & \\
\hline & & 0.999 & 0.732 & 0.177 & 0.732 & 0.999 & & & 0.102 & 0.008 & 1.0 \\
\hline & 1.00 & 1.000 & 1.000 & 0.177 & 1.000 & 1.000 & 000 & 0 & 9 & 1. & 1.000 \\
\hline & & 1.000 & 1.000 & 0.234 & 1.000 & 1.000 & & & & & \\
\hline 50 & 1.50 & 1.000 & 1.000 & 0.177 & 1.000 & 1.000 & 1.000 & & 0.179 & & 0 \\
\hline & & 1.000 & 1.000 & 0.234 & 1.000 & 1.000 & 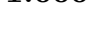 & 2000 & 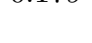 & 19080 & 年 \\
\hline & 200 & 1.000 & 0.976 & 0.177 & 0.976 & 1.000 & 000 & 0.075 & 0179 & & 0 \\
\hline & 2.00 & 1.000 & 1.000 & 0.234 & 1.000 & 1.000 & 1.000 & 0.510 & 0.115 & 0.310 & 1.000 \\
\hline
\end{tabular}

\section{Conclusion}

When SPC is performed for short production runs, the number of inspections is limited. It is impossible for quality practitioner to get a correct estimation of the population parameters $\mu_{0}$ and $\delta_{0}$ for quality characteristic. The $T$ statistic is adopted as a tentative solution to overcome this problem, because the preliminary Phase I estimation of the quality character population parameters is not needed. In this paper, the AEWMA $t$ control chart is proposed. Based on the investigation of the joint effect of parameters $\lambda$ and $\gamma$, a new optimization algorithm is proposed for the statistical design of AEWMA $t$ control chart. Two statistical performance measures have been calculated by implementing the Markov chain theory. Simulations are performed to compare the performance of EWMA $t$ control chart and AEWMA $t$ control chart. The results show that the AEWMA $t$ control chart can overcome the inertia problem of EWMA $t$ control chart. Future research will be focused on the investigation of the evaluation of the economic impact of AEWMA $t$ control chart.

\section{References}

[1] Quesenberry C P. The effect of sample size on estimated limits for $\bar{X}$ and $X$ control charts. Journal of Quality Technology, 1993, 25(4): 237-247.

[2] Neduraman G, Pigniatiello J J. On estimating $\bar{X}$ control chart limits. Journal of Quality Technology, 2001, 33(3): 206-212.

[3] Tsai T R, Lin H C, Wu S J. An alternative control chart approach based on small numbers of subgroups. International Journal of Information and Manufacturing Sciences, 2004, 15(4): 61-73.

[4] Tsai T R, Lin J J, Wu S J, et al. On estimating control limits of $\bar{X}$ chart when the number of subgroups is small. International Journal of Advanced Manufacturing Technology, 2005, 26(11): 1312-1316.

[5] Quesenberry C P. SPC $Q$ charts for star-up processes and short or long runs. Journal of Quality Technology, 1991, 23(3): 213-224. 
[6] Quesenberry C P. On properties for $Q$ charts for variables. Journal of Quality Technology, 1995, 27(3): 184-203.

[7] Castillo E D, Montgomery D C. Short-run statistical process control: $Q$-chart enhancements and alternative methods. Quality and Reliability Engineering International, 1994, 10(2): 87-97.

[8] He F, Jiang W, Shu L. Improved self-starting control charts for short runs. Quality Technology and Quantitative Management, 2008, 5(3): 289-308.

[9] Celano G, Castagliola P, Trovato E, et al. Shewhart and EWMA $t$ control charts for short production runs. Quality and Reliability Engineering International, 2011, 27(3): 313-326.

[10] Zhang L, Chen G, Castagliola P. On $t$ and EWMA $t$ charts for monitoring changes in the process mean. Quality and Reliability Engineering International, 2009, 25(8): 933-945.

[11] Kazemzadeh R B, Karbasian M, Babakhani M A. An EWMA $t$ chart with variable sampling intervals for monitoring the process mean. The International Journal of Advanced Manufacturing Technology, 2013, 66(1-4): 125-139.

[12] Nenes G, Tagaras G. The CUSUM chart for monitoring short production runs. International Journal of Production Research, 2005, 44(8): 1569-1587.

[13] Celano G, Castagliola P, Fichera S, et al. Performance of $t$ control charts in short runs with unknown shift sizes. Computers and industrial Engineering, 2013, 64(1): 56-68.

[14] Celano G, Castagliola P, Trovato E, et al. The economic performance of the shewhart $t$ chart. Quality and Reliability Engineering International, 2012, 28(2): 159-180.

[15] Celano G, Castagliola P, Trovato E. The economic performance of a CUSUM $t$ Control Chart for Monitoring Short Production Runs. Quality Technology and Quantitative Management, 2012, 9(4): 329-354.

[16] Capizzi G, Masarotto G. An adaptive exponentially weighted moving average control chart. Technometrics, 2003, 45(3): 199-207.

[17] Woodall W H, Mahmoud M A. The inertial properties of quality control charts. Technometrics, 2005, 47(4): 425-436.

[18] Shu L J. An adaptive exponentially weighted moving average control chart for monitoring process variances. Journal of Statistical Computation and Simulation, 2008, 78(4): 367-384.

[19] Saleh N A, Mahmoud M A, Abdel-Salam G A-S. The performance of the adaptive exponentially weighted moving average control chart with estimated parameters. Quality and Reliability Engineering International, 2013, 29(4): 595-606.

[20] Yashchin E. Estimating the current mean of a process subject to abrupt changes. Technometrics, 1995, 37(3): 311-323.

[21] Su Y, Shu L, Tsui K L. Adaptive EWMA procedures for monitoring processes subject to linear drifts. Computational Statistics and Data Analysis, 2011, 55(10): 2819-2829. 